\title{
Evolutionary Hypernetworks based Radio Spectrum Profiling in Cognitive Radio Ad hoc Networks
}

\author{
Shah Nawaz Khan and Andreas Mitschele-Thiel \\ Integrated Communication Systems Group, Technische Universität Ilmenau \\ P.O. Box 100 565, 98693 Ilmenau, Germany \\ Email: shah-nawaz.khan@tu-ilmenau.de
}

\begin{abstract}
In this paper we present a novel idea of evolutionary hypernetworks based radio spectrum profiling for Cognitive Radio (CR) ad hoc networks. Spectrum profiling enables CR nodes to abstract and predict secondary channel access opportunities from primitive spectrum sensing data. The abstracted information can be used for channel characterization which facilitates better channel selection and mobility in CR nodes. We use Evolutionary Hypernetworks for realizing the proposed concept which can also be viewed as a distributed network support architecture. We present the main concepts, their application to $C R$ ad hoc networks, and an analysis of its impact on overall network performance. The results indicate that hypernetwork based radio spectrum profiling achieves considerable performance advantages over existing approaches.
\end{abstract}

Keywords-Cognitive radio, Spectrum profiling, Channel prediction

\section{INTRODUCTION}

Radio spectrum is a limited and valuable resource considering the overall picture of existing wireless communication systems. However, this resource has not been utilized in the most efficient way which has resulted in spectrum scarcity and underutilization problems. Realization of these problems have resulted in the formulation of opportunistic and dynamic spectrum access concepts which are generally discussed in conjunction with Cognitive Radio (CR) networks. CR networks are considered to be able to identify and utilize any radio spectrum opportunities provided by the existing wireless networks. In the existing literature, spectrum sensing is considered as the Eyes and Ears of a CR node through which it can identify spectrum holes and avoid interference with the so called Primary Users $(P U)$. These tasks are generally considered to be interrelated but they have very different timing requirements. Identify spectrum holes requires monitoring of the radio spectrum for a longer period of time whereas interference avoidance with PUs should be instantaneous. Spectrum sensing is also the enabler for flexible Software Defined Radio (SDR) based medium access control protocols which have stringent time constraints as well. In the traditional 802.11 family of protocols for example, the basic channel access tasks are in the scale of microseconds. If a similar functionality is assumed of SDR based MAC protocols, the spectrum sensing should be equally fast. It can be concluded that for avoiding interference, the reliability and time-efficiency of spectrum sensing is very important. For identifying spectrum holes however, such primitive results are not sufficient. There needs to be an abstraction process that can characterize spectrum holes from sensing results. Furthermore, the identified spectrum holes must be quantified to determine whether they can fulfill the SU applications' requirements. This serves as the basis for our proposed Hypernetworks based Radio Spectrum Profiling concept which aims to characterize the radio spectrum according to the application requirements of the CR nodes. This concept is specially important for CR ad hoc networks which heavily rely on spectrum sensing and have no infrastructural support. The contributions of this paper in the outlined context can be summarized as follows:

- Introduction of a new radio spectrum profiling concept for channel characterization,

- $\quad$ Application of Evolutionary Hypernetworks for radio spectrum profiling,

- Presentation of a new channel state prediction algorithm based on hypernetworks that can tolerate miss detection and false alarms and

- Analysis of CR network performance with the new hypernetworks based spectrum profiling concept.

The rest of the paper is organized as follows. Section II addresses the related work on spectrum characterization and channel prediction. An introduction to Hypernetworks is given in Section III before the details of the proposed evolutionary hypernetworks based spectrum profiling in Section IV. Simulation results are provided in Section $\mathrm{V}$ and the paper is concluded with a summary and future work in Section VI.

\section{RELATED WORK}

To the best of our knowledge, the proposed hypernetworks based radio spectrum profiling concept is novel for CR ad hoc networks. However, a number of research articles addressing channel prediction, channel recommendation and secondary access can be considered as related work since the hypernetworks based spectrum profiling aims to achieve similar objectives. Prediction techniques in CR networks have been applied for of PU activity and channel behavior prediction. The authors in [1] survey the main approaches applied in literature for channel prediction in CR context. They overview the main approaches and classify them based on the prediction techniques used including Hidden Markov Models (HMM), Multilayer Perceptron Neural Networks, Bayesian Inference, Autoregressive Model, and Moving Average based prediction. As the results of channel prediction can be applied to the optimization of different CR functions, no comparative analysis of these techniques has been presented. The application of HMM to predict the basic state transitions involved in ON/OFF PU channel usage model can be found in many articles. 
The authors in [2] present a binary time series approach to spectrum prediction in CR networks. They apply HMM to predict the next state(s) of the channel based on the historic data. They essentially predict the next values of spectrum sensing and relate them to spectrum holes. The authors in [3] also apply HMM based prediction technique for multi-stepahead prediction. They aim to avoid interference with PUs based on the results of the prediction. They measure the level of interference caused by CR network and propose to keep it to a predefined level. Similar technique is used by authors in [4] to evaluate the radio resource availability in 802.11 networks scenario and apply multi-step-ahead prediction derived through an auto-regression (AR) Model. They apply their technique to 802.11 network data traffic by measuring the radio resource availability through Network Allocation Vector (NAV). HMM based approach has also been used by authors in [5] to predict exponentially distributed PU activity over radio spectrum. For most of the HMM based approaches, the activities of the channel are modeled under Markovian assumptions. The authors in [6] however, present temporal spectrum sharing scheme based on PU activity prediction that considers bursty PU traffic whose characteristics are not captured effectively by Markovian process. They propose to adapt the SU transmission power levels that can be adapted to any source traffic model of PUs. The benefits of PU activity prediction have been shown to optimize different functions of a CR node. The authors in [7] show the application of fast discovery of spectrum opportunity in multichannel context to $\mathrm{CR}$ performance optimization. They propose an adaptive sensing period optimization algorithm together with an optimal channel-sequencing algorithm. This allows a CR node to find spectrum opportunities from a number of available channels efficiently without loosing significant spectrum opportunities provided by the considered radio spectrum. They also show that the channel discovery delay can be reduced to less than half a second with an optimized channel sensing and sequencing approach. The same prediction algorithm has been applied for proactive channel access in [8] in order to vacate a channel before the PU arrives. They essentially apply the results from [7] to a different optimization objective. The information about channels derived through spectrum sensing has also been considered for CR optimization outside the context of prediction. The authors in [9] present a channel recommendation framework in which distributed $\mathrm{CR}$ nodes complement each other's channel access by recommending a successfully used channel. They derive the inspiration from customer reviews system associated with major online retail systems. The same idea has been optimized in [10] where the authors formulate the problem as an average reward based Markov decision process. They compare the performance of a dynamic spectrum access system using the adaptive recommendation system with a static channel recommendation system and show performance benefits of upto $15 \%$. Existing literature on channel characterization and prediction generally assumes the spectrum sensing to be 100 percent accurate. This assumption is made in order to have a realistic representation of PUs communication in a binary time series. It is well known however, that even the most sophisticated spectrum sensing algorithms are prone to miss-detections and false alarms. This renders the algorithms that predict next state(s) of binary time series prone to lower performance when considered in a realistic CR network scenario. Another assumption that is explicitly made or implied is that zero bits in binary time series represent spectrum holes for secondary access. Based on the discussion in Section I, this assumption also does not hold. If the spectrum sensing results are collected in the scale of microseconds, a single zero bit loses its significance for overall spectrum hole representation and gains it for SDR based MAC protocols. Our proposed spectrum profiling concept therefore, does not rely on such assumptions and instead, applies an abstraction process that is independent of the accuracy and time scale of spectrum sensing results.

\section{HYPERNETWORKS}

Hypernetworks is a relatively new research domain and a candidate architecture for cognitive learning and memory [11]. It is a graphical model that can abstract both low and high level of interactions among elements of a dataset. Hypernetworks are an extension of hypergraphs. A hypergraph is an undirected graph $G$, the edges of which can connect any number of non-null vertices. Formally, $G=\{X, E\}$, where $X=\left\{X_{1}, X_{2}, \ldots X_{n}\right\}, E=\left\{E_{1}, E_{2}, \ldots E_{m}\right\}$ and $E_{i}=$ $\left\{x_{i_{1}}, x_{i_{2}}, \ldots, x_{i_{k}}\right\}$. The edges of a hypergraph $E_{i}$ are referred to as Hyperedges. Each hyperedge which is synonymous to a non-empty set, encapsulates some primitive relation in the dataset. The number of elements $k$ encapsulated in a hyperedge representing its cardinality, is referred to as a $k$-hyperedge. Hypernetworks are a generalization of hypergraphs which assign a particular weight to the hyperedges represented by the width of the hyperedge. The more stronger a relation in the dataset, the larger the width of the hyperedge. Formally, a hypernetwork is a triple $H=(X, E, W)$ where $X$ represent the set of vertices or elements of the data set, $E$ represents the set of hyperedges, and $W$ represents the set of weights associated with each hyperedge showing its strength in the dataset. The cardinality of a hyperedge is referred to as the order of a hyperedge. From a given dataset $D=\left\{x^{(n)}\right\}_{n=1}^{N}$ of $N$ example patterns, the hypernetwork represents the probability of generating $D$ :

$$
P(D \mid W)=\prod_{n=1}^{N} P\left(x^{(n)} \mid W\right)
$$

The $W$ term in Equation 1 represents both the weight of the hyperedge and its structure. With hyperedges, both low and high level features of the dataset can be encapsulated into the memory of hypernetwork. The varying order of the hyperedges allows hypernetworks to keep a large number of random memory fragments $x_{i_{1}}^{(n)} x_{i_{2}}^{(n)} \ldots x_{i_{k}}^{(n)}$ to estimate the probability of any particular fragment. The probability of an individual fragment or pattern can be given as [11]:

$$
\begin{gathered}
P\left(x^{(n)} \mid W\right)=\frac{1}{Z(W)} \exp \left[\sum_{k=1}^{K} \frac{1}{C(k)} \times\right. \\
\left.\sum_{i_{1}, i_{2}, \ldots i_{k}} w_{i_{1}, i_{2}, \ldots i_{k}}^{(k)} x_{i_{1}}^{(n)} x_{i_{2}}^{(n)} \ldots x_{i_{k}}^{(n)}\right]
\end{gathered}
$$

where $Z(W)$ is a normalizing term and $C(k)$ is the number of possible hyperedges of order $k$. The number of possible patterns or fragments grows exponentially and therefore an evolutionary approach of selection, replacement, and reinforcement 
towards finding an appropriate ensemble of hyperedges can be applied. Related work on hypernetworks have demonstrated its ability to predict future states from previous observations as well as its ability to mimic artificial intelligence [12], [13], [14]. Hypernetworks have also been compared to other approaches of learning and prediction and the results have shown hypernetworks to be comparable in overall achievable results [15].

\section{HyPERNETWORKS BASED SPECTRUM PROFILING}

We coin the term Cognitive Radio Spectrum Profiling as the process of abstracting usable channel information from primitive spectrum sensing performed by all $\mathrm{CR}$ nodes. The abstracted information can then be used to optimize the performance of $\mathrm{CR}$ nodes in terms of their channel access and mobility as well as for the development and support of a distributed network support architecture [16]. Evolutionary hypernetworks are well suited to the realization of radio spectrum profiling concept for CR ad hoc networks. We apply evolutionary hypernetworks in CR context for the abstraction and prediction of spectrum holes from spectrum sensing data. Hypernetworks can achieve these objectives based on the requirements of secondary user applications represented through hyperedges. Each hyperedge encapsulates a pattern of interest in the dataset for specific application requirements. Over the course of hypernetwork learning and evolution, the hyperedges are either reinforced or discarded based on their relevance in the original dataset.

\section{A. Hypernetwork Initialization}

For simplicity, we shall consider the hypernetwork based spectrum profiling on a single CR node. The hypernetwork initialization/creation process is depicted in Fig. 1. From the basic spectrum sensing results data, with an acceptable level of errors due to miss-detection and false-alarms, the hypernetwork first abstracts the patterns of interest. A pattern is a variable sequence of bits ( 2 or more) in the binary timeseries data. These patterns are selected based on the minimum requirements of the secondary user application. In this paper, we classify the patterns based on the minimum unit of time a PU channel should be free to fulfill the QoS requirements of a secondary user application. In CR context, the patterns are essentially variable-length sequences of zeros implying the durations of no PU channel activity and ones implying busy channel states. The use of variable length patterns allows hypernetworks to abstract and predict different levels of PU activities on a channel. Assuming that miss-detections and falsealarms are not sequentially redundant, each pattern can ignore some incorrect bits in the overall pattern by replacement. In Fig. 1 for example, pattern P1 can ignore the existence of bit ' 1 ' in the sequence and treat the whole pattern as a sequence as zeros. This makes sense especially when the significance of a single bit is reduced by the minute time-scale of spectrum sensing. This aspect is highlighted in Fig. 2 which shows the time required to opportunistically transmit $1 \mathrm{MB}$ of data over two GSM channels having fixed PU duty cycles of 70 and 40 percent respectively. The $\mathrm{x}$-axis shows the same duty cycle mapped to different time scales. When the PUs are off (point 0 on $\mathrm{x}$-axis), the throughput achieved is almost equal to the possible maximum on a $200 \mathrm{Khz}$ GSM channel. When the PUs

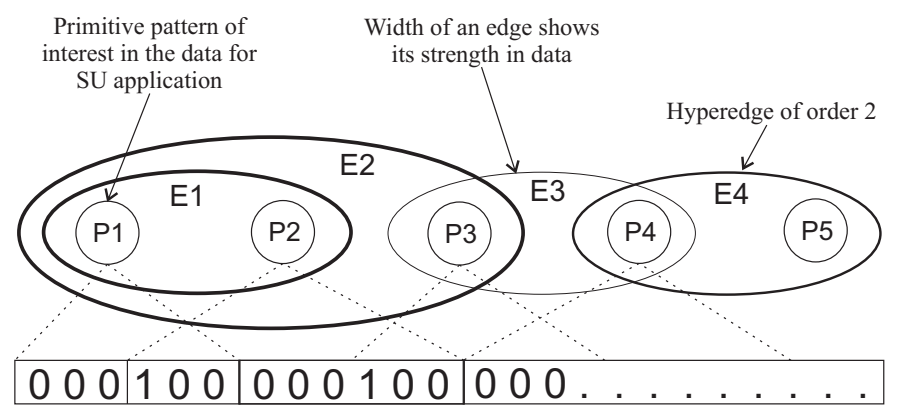

Fig. 1: Hypernetwork with 4 hyperedges and 5 patterns

operate at varying time-scales, the throughput drops considerably. When the PU provides spectrum access opportunity in the order of a few milliseconds, the throughput drops to zero as no secondary user connection can be established in such a short channel idle phase. From this result it is clear that a spectrum opportunity provided in the scale of microseconds is not suitable for any secondary application and therefore there is no significance of the sensing results in that time-scale. An opposite argument could be that the spectrum sensing duration can be increased but this creates two problems. One, the time scale on which radio and MAC protocols operate (microseconds in existing networks) requires the sensing functions to be equally fast. Second, the decision accessing a spectrum hole must be taken at the very beginning of that opportunity and not after long observation. The initial pattern creation for hypernetworks can compensate for these problems where only information of interest is abstracted from the spectrum sensor data. The patterns also serve to smooth out some of the spectrum sensing fluctuations that may be caused by temporal variations in the RF environment. Furthermore, since different applications require different levels of channel access guarantees, the initial pattern creation process serves to classify the channels accordingly. The abstracted list of patterns serves as the basic input to the hypernetwork which randomly creates hyperedges from these patterns. The hypernetwork in Fig. 1 for example, has four hyperedges formed from five basic patterns. The number of possible hyperedges in the hypernetwork depends upon the specified minimum and maximum order of the hyperedges and grows exponentially. The patterns encapsulated inside a hyperedge are ordered based on their abstraction from the spectrum sensing data. In other words, each hyperedge encapsulates a sequence of observations on a pre-defined channel. The hyperedge creation process is undertaken using random sampling of the distinct vertices i.e. patterns. The parameters that control the complexity of the hypernetworks is the min and max order to the hyperedges. If $\min =\max$ then a fix order hypernetwork is created where each hyperedge encapsulates the same number of sequential patterns. If $\min \neq \max$ then a mixed order hypernetwork is created where small order hyperedges encapsulate small memory fragments and higher order hyperedges encapsulate larger, more specific channel activity information. The initial weight of a hyperedge is set to the same value i.e. 1, unless otherwise specified at the time of initialization. Explicit initial weights can be assigned to certain types of hyperedges in order to emphasize the importance of that particular relationship in the dataset. For example, a hyperedge encapsulating the 


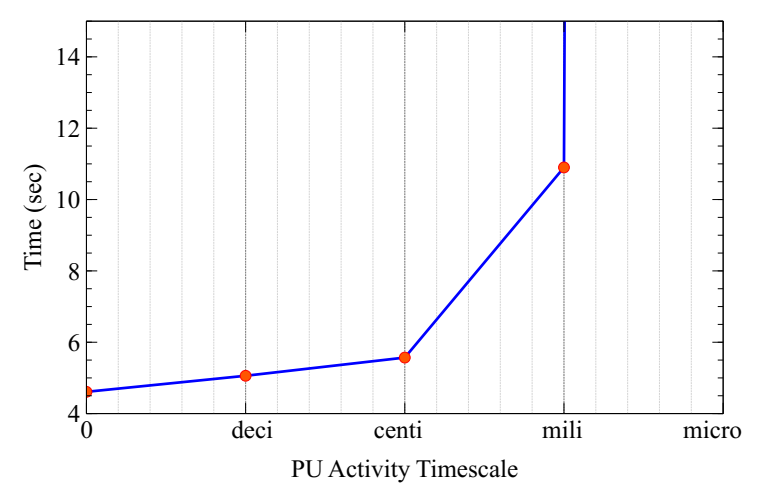

Fig. 2: Throughput achieved over 2 GSM channels with varying time-scales of PU activity

patterns associated with PU connection and transmission phase can be assigned a higher weight in the hypernetwork.

\section{B. Evolutionary Learning Process}

The hypernetwork learning is an iterative process through which it evolves and assigns different weights to the hyperedges. The assigned weights represent the relative frequency of that pattern in the dataset. The number of iterations is a parameter of the algorithm and can be set at the beginning of learning process. In the classical hypernetworks, the set of randomly sampled hyperedges remain alive throughout the lifetime of the learning process. In evolutionary hypernetworks however, the addition of new as well as removal of weak/old hyperedges is possible. On every iteration $j$, a new hyperedge $E^{\prime}$ of order $k \in\{\min , \max \}$ is created. Since the new hyperedge is created from the same patterns pool, the encapsulated elements can be matched to the previously sampled hyperedges. The weight of the matched hyperedge $w_{E}$ is increased by a reward function for the next iteration $j+1$ :

$$
w_{E}^{j+1}=w_{E}^{j}+\delta\left(E, E^{\prime}\right)
$$

where $\delta$ is a reward function. The reward function used is similar to that in [15] which bases the reward on the order of a hyperedge. For the reward function, the last pattern in the hyperedge is called its $\operatorname{tag} t_{E}$. The tag of the hyperedge is important as it is used for the prediction purpose later. The tag of the hyperedge can be the last element(s) of the encapsulated pattern. If $E$ matches $E^{\prime}$ along with respective tags, the reward is equal to the order of $E$. If the tags do not match, a penalty is imposed instead which is equal to the negative of the order of E. Formally,

$$
\begin{aligned}
& \delta\left(E, E^{\prime}\right) \\
& \quad=\left\{\begin{aligned}
k, & \forall_{i} \in\left[1, k^{\prime}\right], \exists_{j} \in[1, k]: e_{i}^{\prime}=e_{j} \wedge t_{E}=t_{E}^{\prime} \\
-k, & \forall_{i} \in\left[1, k^{\prime}\right], \exists_{j} \in[1, k]: e_{i}^{\prime}=e_{j} \wedge t_{E} \neq t_{E}^{\prime} \\
0, & \text { otherwise }
\end{aligned}\right.
\end{aligned}
$$

where $E$ is a $k$ order hyperedge with tag $t_{E}$, and $E^{\prime}$ is the new hyperedge with tag $t_{E}^{\prime}$. In the classical hypernetworks, the number of randomly sampled hyperedges is usually kept

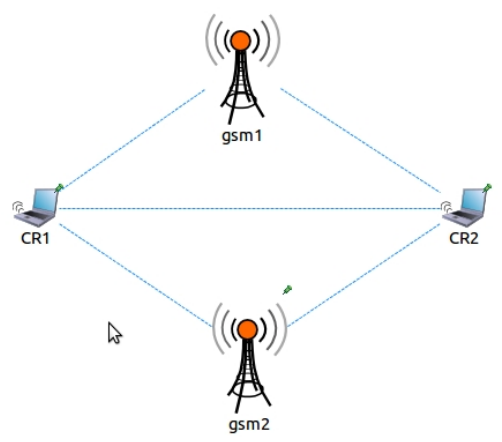

Fig. 3: Simulated network scenario

very high in order to cover most of the search space from the dataset. The initial abstraction of binary time series into patterns of interest allows to keep this complexity under bounds. Furthermore, we employ the Data-driven Evolutionary Training approach [15] to optimize the learning process. When a newly generated hyperedge from the pattern pool is not matched with any of the existing sampled hyperedges, one of the smallest weight hyperedge is replaced with the newly created hyperedge from the dataset. This allows for a continuous exploration of search space while keeping the hypernetwork information relevant to desired objectives. If the newly added information is relevant, its weight will increase in future iterations otherwise it will be discarded through the evolutionary process. To summarize, hyperedges encapsulate different levels of secondary channel access opportunities through mix-order hypernetwork. During the evolutionary learning process, the relevant information in the hyperedges is reinforced that can serve to characterize channels and optimize CR node functions.

\section{Hypernetworks based Prediction}

Once the hypernetwork is trained over the pattern pool, the desired information is reflected in the developed structure of the hypernetwork and the forecasting process is straightforward. The most frequent patterns in the dataset are reflected in the weights of their respective hyperedges. The patterns that are least frequent in the dataset have very weak hyperedges. The structure of the hypernetwork also represents the different parameters set for the initialization and training i.e. min-max order of the hyperedges, evolution and iterations. When a new pattern extracted from spectrum sensing data is given as input to the trained hypernetwork it is first matched to the candidate hyperedges. The input pattern is always a subset of the patterns encapsulated inside the hyperedges. The hypernetwork predicts the tag of the highest weight hyperedge that matches the input pattern. The prediction of a future pattern is of higher significance to a CR application than the prediction of spectrum sensing results considered in many related literature. If the tag of the hyperedge is a representation of the absence of PU for a specific time frame, the channel access can be initialized by a CR transmitter.

\section{Simulation Analysis}

In order to analyze the effectiveness of the hypernetworks based spectrum profiling in a CR ad hoc networks, we carried out system level simulations using crSimulator [17]. As 
TABLE I: Hypernetwork parameter settings

\begin{tabular}{|l|c|}
\hline Parameter & Value \\
\hline Order $\min$ & 3 \\
\hline Order $\max$ & 6 \\
\hline Patterns $\max$ & 3 \\
\hline Reward function & $\delta$ \\
\hline PU Activity & Variable Duty Cycle $(30-75 \%)$ \\
\hline Dataset size & 100 \\
\hline
\end{tabular}

TABLE II: Successful forecasts

\begin{tabular}{|l|c|c|}
\hline Setup & Avg Order & Success Rate \\
\hline $\operatorname{EHN}(3,6) \delta$ & 4.4 & $74 \%$ \\
\hline $\operatorname{EHN}(3,5) \delta$ & 4 & $71 \%$ \\
\hline $\operatorname{EHN}(3,4) \delta$ & 3.4 & $71 \%$ \\
\hline $\operatorname{EHN}(3,3) \delta$ & 3 & $68 \%$ \\
\hline
\end{tabular}

a multi-hop CR ad hoc network can be considered as an extension of many single-hop links, we analyze the effect of hypernetwork based spectrum profiling on a single multichannel CR link. The evaluated network scenario is presented in Fig. 3 where two CR nodes attempt to use two GSM specification channels opportunistically. It should be noted, that the selection of GSM channels for the evaluation over any other spectrum band is of little significance. The important aspect is the representation of accurate channel state based on PU activity profiles and the existence of secondary access opportunities. Representation of realistic PU activity profiles is an active area of research in itself as it is very difficult to have a generalized model for different locations and spectrum bands. From the numerous spectrum measurement campaigns, it has been observed that the PU activity can scale from no activity such as in TV broadcast bands, to $100 \%$ utilization of spectrum such as in GSM or WiFi bands depending upon time and location [18]. In literature, two extremes can be found for PU activity modeling which are using a fixed pattern with defined duty cycle and fully stochastic behavior with different distributions. The actual PU activity distribution in any particular location can be assumed to lie somewhere in the middle of these two extremes. In order to evaluate the performance of the hypernetworks based radio spectrum profiling, we employ the binary (ON/OFF) model for PU activity with time varying duty-cycles (30 to $70 \%$ ) during the course of simulation time [19].

\section{A. Successful forecasts}

Table I shows the settings used for the formation and training of the hypernetworks. The spectrum sensing results are abstracted into three distinct patterns based on the requirements of two different secondary user applications. The first two patterns abstract two distinct spectrum access opportunities while the third pattern represents a busy channel state. With these three basic patterns, any binary time series data of spectrum sensing can be abstracted and fed into a hypernetwork structure. This process essentially transforms binary data into time series of the three primitive patterns the association among which is learned by hypernetwork through an iterative evolution process. Table II gives the results of the forecasting process. The notation $\operatorname{EHN}(3,6) \delta$ denotes an evolutionary

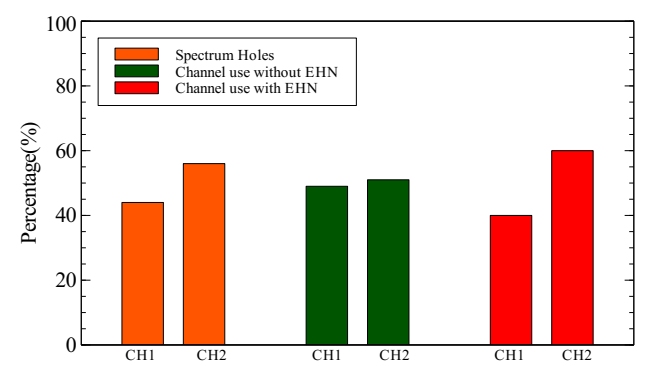

Fig. 4: Percentage of spectrum opportunities on two PU channels and their opportunistic usage

hypernetwork with $\min -\max$ order of 3-6 of the hyperedges and a reward function of $\delta$. The success rates shown for the different order hypernetworks are for test cases where similarity between training patterns and and test patterns was upto $60 \%$ only. If certain patterns of activity on PU channels repeat to a higher degree, the success rates of hypernetworks also increase proportionally. The success rates achieved through hypernetworks based channel state forecasting are significant and can potentially be optimized further by using dynamic approach to hypernetwork training and evolution.

\section{B. Channels characterization}

The hypernetworks based radio spectrum profiling can characterize available channels based on application requirements. To test this, a time variant duty cycle between 60 to $70 \%$ was applied to the network scenario of Fig. 3 where each PU (GSM nodes in Fig. 3) followed its independent timescale of transmission. In a scenario like this, the CR nodes will attempt to access both channels with equal probability without any profiling of the channels. With hypernetworks based radio spectrum profiling however, the CR nodes check for the probability of the next pattern in the channel and try to access that channel which has a predicted pattern of interest as forecast. This effect is shown in Fig. 4 which shows the distribution of spectrum opportunities during the simulation on both PU channels and their utilization by CR nodes. The hypernetworks based profiling enables $\mathrm{CR}$ nodes to access the suitable channel $(\mathrm{CH} 2)$ more frequently. This result shows that CR network performance can be optimized by differentiating among channels that generally look to provide similar spectrum access opportunities. The performance benefit increase even more when there is a clear difference between the channels as shown in Fig. 5. This performance improvement can also be seen in the number of channel handovers performed by CR network during the simulation and the achieved throughput as shown in Fig. 6. For the analysis of the impact on handovers, we assumed that the channel switching does not incur considerable time overhead. In real scenarios however, performing spectrum handovers will require some pre-agreement, sharing of knowledge about back-up channels through control message exchange, or certain policies which may incur considerable time and energy costs.

\section{CONClusion AND Future Work}

In this paper we presented a novel radio spectrum profiling framework that is based on evolutionary hypernetworks. Hy- 


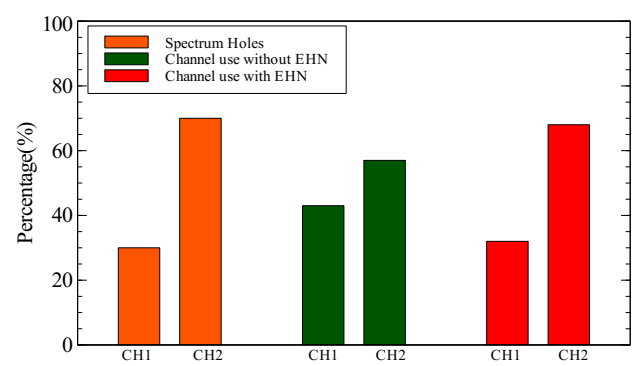

Fig. 5: Secondary channel access with clear differentiation between channels

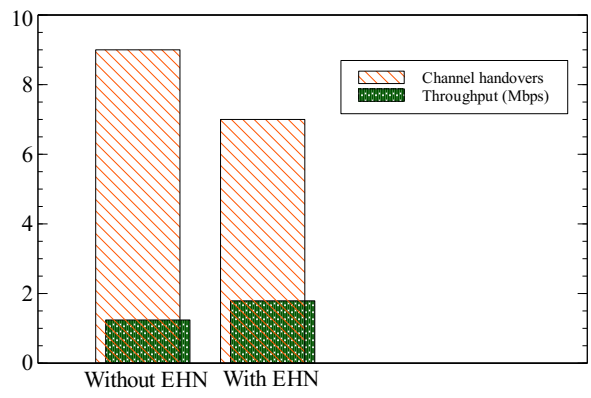

Fig. 6: Achieved throughput and performed handovers

pernetworks can effectively capture and abstract the patterns of interest from the primitive spectrum sensing data and enable characterization of the available channels based on secondary users' application requirements. It was shown that spectrum holes can be identified from primitive binary time series of channel states and utilized for differentiating among available channels. In contrast to other channel prediction techniques based on HMM, the hypernetworks do not require the spectrum sensing data to be 100 percent accurate and can reliably forecast the future channel states. Hypernetworks are suited to identifying spectrum opportunities for different types of applications. The implicit data smoothing through formation of patterns in the primitive sensing data also serves to mitigate the effect of minor channel fluctuations in RF environment. Currently, a hypernetwork learns from a single dataset which implies that only one channel/band can be abstracted and multiple instances are required for multiple channels. A future extension could be to analyze cross-channel pattern creation for a single hypernetwork especially for adjacent frequency channels. This paper provided the analysis of hypernetworks for a single link CR network where the environment was similar for both transmitter and receiver nodes. However, in a distributed ad hoc network, disagreements about channel states among network peers will happen and are subject to further investigation.

\section{ACKNOWLEDGMENT}

This work is being carried out within the scope of the International Graduate School on Mobile Communications (Mobicom) at Ilmenau University of Technology, supported by the German Research Foundation (GRK1487) and the Carl Zeiss Foundation, Germany.The authors would like to further acknowledge Dr. Elena Bautu at Ovidius University, Constanta Romania, for her guidance, help and support.

\section{REFERENCES}

[1] X. Xing, T. Jing, W. Cheng, Y. Huo, and X. Cheng, "Spectrum prediction in cognitive radio networks," Wireless Communications, IEEE, vol. 20, no. 2, pp. 90-96, 2013.

[2] S. Yarkan and H. Arslan, "Binary time series approach to spectrum prediction for cognitive radio," in Vehicular Technology Conference, IEEE 66th, 2007, pp. 1563-1567.

[3] R. Min, D. Qu, Y. Cao, and G. Zhong, "Interference avoidance based on multi-step-ahead prediction for cognitive radio," in Communication Systems, 11th IEEE Singapore International Conference on, 2008, pp. 227-231.

[4] S. Kaneko, S. Nomoto, T. Ueda, S. Nomura, and K. Takeuchi, "Predicting radio resource availability in cognitive radio - an experimental examination," in Cognitive Radio Oriented Wireless Networks and Communications, 3rd International Conference on, 2008, pp. 1-6.

[5] C.-H. Liu, W. Gabran, and D. Cabric, "Prediction of exponentially distributed primary user traffic for dynamic spectrum access," in Global Communications Conference (GLOBECOM), IEEE, 2012, pp. 14411446.

[6] K. W. Sung, S.-L. Kim, and J. Zander, "Temporal spectrum sharing based on primary user activity prediction," Wireless Communications, IEEE Transactions on, vol. 9, no. 12, pp. 3848-3855, 2010.

[7] H. Kim and K. Shin, "Efficient discovery of spectrum opportunities with mac-layer sensing in cognitive radio networks," Mobile Computing, IEEE Transactions on, vol. 7, no. 5, pp. 533-545, 2008.

[8] L. Yang, L. Cao, and H. Zheng, "Proactive channel access in dynamic spectrum networks," Physical Communication, vol. 1, no. 2, pp. 103 111, 2008.

[9] X. Chen, J. Huang, and H. Li, "Adaptive channel recommendation for dynamic spectrum access," in IEEE Symposium on New Frontiers in Dynamic Spectrum Access Networks (DySPAN), May 2011.

[10] H. Li, "Customer reviews in spectrum: Recommendation system in cognitive radio networks," in New Frontiers in Dynamic Spectrum, IEEE Symposium on, april 2010, pp. 1-9.

[11] B.-T. Zhang, "Hypernetworks: A molecular evolutionary architecture for cognitive learning and memory," Computational Intelligence Magazine, IEEE, vol. 3, no. 3, pp. 49-63, 2008.

[12] J.-K. Kim and B.-T. Zhang, "Evolving hypernetworks for pattern classification," in Evolutionary Computation, IEEE Congress on, 2007, pp. $1856-1862$.

[13] H.-W. Kim, B.-H. Kim, and B.-T. Zhang, "Evolutionary hypernetworks for learning to generate music from examples," in Fuzzy Systems, IEEE International Conference on, 2009, pp. 47-52.

[14] S. Kim, S.-J. Kim, and B.-T. Zhang, "Evolving hypernetwork classifiers for microrna expression profile analysis," in Evolutionary Computation, IEEE Congress on, 2007, pp. 313-319.

[15] E. Bautu, S. Kim, A. Bautu, H. Luchian, and B.-T. Zhang, "Evolving hypernetwork models of binary time series for forecasting price movements on stock markets," in Evolutionary Computation, IEEE Congress on, 2009, pp. 166-173.

[16] S. N. Khan, M. A. Kalil, and A. Mitschele-Thiel, "Distributed resource map: A database-driven network support architecture for cognitive radio ad hoc networks," in Ultra Modern Telecommunications and Control Systems and Workshops (ICUMT), 4th International Congress on, 2012, pp. 188-194.

[17] S. Khan, M. A. Kalil, and A. Mitschele-Thiel, "crsimulator: A discrete simulation model for cognitive radio ad hoc networks in omnet++," in Wireless and Mobile Networking Conference (WMNC), 6th Joint IFIP, 2013, pp. 1-7.

[18] L. Stabellini, "Quantifying and modeling spectrum opportunities in a real wireless environment," in Wireless Communications and Networking Conference (WCNC), IEEE, 2010, pp. 1-6.

[19] M. Lopez-Benitez and F. Casadevall, "Empirical time-dimension model of spectrum use based on a discrete-time markov chain with deterministic and stochastic duty cycle models," Vehicular Technology, IEEE Transactions on, vol. 60, no. 6, pp. 2519-2533, 2011. 